\title{
Complex interventions and the chamber of secrets: understanding why they work and why they do not
}

\author{
Shaun Treweek
}

$J$ R Soc Med 2005;98:553

Harry Potter's magic touch is notably absent from the bullets used to achieve professional behaviour change. ${ }^{1}$ Healthcare professionals want to provide high quality care but may be unwilling or unable to change their behaviour. Maybe they are sceptical about the need for change, or practice routines make change difficult. Perhaps the nice man from the drug company said something else and he came with free pens to back up his argument. Complex interventions try to grasp these nettles by having several components, which work together to reinforce the change. Despite this, most complex interventions achieve between only $10 \%$ and $20 \%$ change, and many programmes result in no change at all. ${ }^{2}$

Although systematic reviews can tell us what works, they say little about why. Why do some implementations of an intervention (audit, say) lead to change while others do not? How do intervention components work together? With unlimited resources we could probably do enough trials to provide entirely empirical answers to these questions. The problem is that resources are anything but unlimited.

Science has a shortcut: theory. The NASA Deep Impact spacecraft, for example, was not sent in a direction selected because several other spacecraft sent in that direction had hit something and, well, it might work again. Astronomical observation and previous experiments were combined with theory to figure out where comet Tempel 1 was going and where the spacecraft had to be sent for the two to meet. It was an experiment done within a theoretical framework. Much, perhaps most, quality improvement work is not done within an explicit theoretical framework. For example, less than $10 \%$ of studies included in a recent review of guideline implementation ${ }^{3}$ gave a theoretical rationale for the intervention ${ }^{4}$. Doing this would allow researchers to better predict and interpret results, and would inform discussions of generalizability.

Research Fellow, Tayside Centre for General Practice, University of Dundee, Kirsty Semple Way, Dundee DD2 4BF, Scotland, UK

E-mail: s.treweek@chs.dundee.ac.uk
Unlike those planning to lob washing machine-sized objects at comets, individuals designing healthcare complex interventions have a difficult task when selecting their theoretical framework. Like a good sweetshop, the problem is too much choice rather than too little. Michie and colleagues found 33 theories with 128 explanatory constructs $^{5}$, enough to scare the bejesus out of anyone who does not hold a postgraduate qualification in psychology. But if we aim to change professional behaviour can we really afford to ignore theories of human behaviour on the grounds that they are hard to understand? I think not. Find a psychologist, get him or her on the team and then start to argue about the evidence base for these theories but do not ignore them. Other disciplines may also have much to contribute; I fancy that evolutionary biology can offer insights, as can optimization techniques from engineering.

Using complex interventions to change professional behaviour is currently a hit and miss affair and is likely to remain so for as long as interventions are developed without an explicit theoretical framework. A valid framework may not yet exist. But if we want to increase our understanding of how and why complex interventions work we need less magic and more science. Sorry Harry.

\section{REFERENCES}

1 Oxman AD, Thomson MA, Davis DA, Haynes RB. No magic bullets: a systematic review of 102 trials on interventions to improve professional practice. Can Med Assoc J 1995;153:1423-31

2 Grimshaw JM, Shirran L, Thomas R, et al. Changing provider behavior: an overview of systematic reviews of interventions. Med Care 2001;39 (suppl 2):II2-45

3 Grimshaw JM, Thomas RE, MacLennan G, et al. Effectiveness and efficiency of guideline dissemination and implementation strategies. Health Technol Assess 2004;8:1-72

4 Davies P, Walker A, Grimshaw J. Theories of behavior change in studies of guideline implementation. Proc Br Psychol Soc 2003;11:120

5 Michie S, Johnston M, Abraham C, Lawton R, Parker D, Walker A, on behalf of the Psychological Theory Group. Making psychological theory useful for implementing evidence based practice: a consensus approach. Qual Saf Health Care 2005;14:26-33 [doi: 10.1136/ qshc.2004.011155] 